\title{
A Targeted Survey for Scrapie in Jos Plateau State, Nigeria
}

\author{
O. O. Nwankiti, ${ }^{1}$ E. I. Ikeh, ${ }^{2}$ O. A. Arowolo, ${ }^{1}$ A. J. Nwankiti, ${ }^{1}$ \\ M. O. Odugbo, ${ }^{1}$ and T. Seuberlich ${ }^{3}$ \\ ${ }^{1}$ National Veterinary Research Institute, PMB 1 Vom, Plateau State 930010, Nigeria \\ ${ }^{2}$ Department of Medical Microbiology, School of Medicine, University of Jos, PMB 2084, Jos 930001, Nigeria \\ ${ }^{3}$ NeuroCentre, National and OIE Reference Laboratories for BSE and Scrapie, Division of Neurological Sciences, \\ Vetsuisse Faculty, University of Berne, 3001 Berne, Switzerland
}

Correspondence should be addressed to O. O. Nwankiti; obee_nwankiti@yahoo.com

Received 9 May 2013; Accepted 11 July 2013

Academic Editor: Vito A. Vetrugno

Copyright (C) 2013 O. O. Nwankiti et al. This is an open access article distributed under the Creative Commons Attribution License, which permits unrestricted use, distribution, and reproduction in any medium, provided the original work is properly cited.

Scrapie, a disease of sheep and goats with a progressive course and fatal outcome, has not been identified in Nigeria. Anecdotal scrapie reports by livestock workers abound. Livestock diseases like scrapie form huddles in livestock economics of countries. For 8 months we surveyed for scrapie targeting emergency/casualty slaughter sheep and goats in Jos, Nigeria. We clinically examined 510 sheep and 608 goats of local breeds, aged from 12 months to 5 years. In total 31 (5.10\%) goats and no sheep were clinically suspicious for scrapie. Caudal brainstem tissues of suspect animals collected postmortem were analyzed for the disease specific form of the prion protein, $\mathrm{PrP}^{\mathrm{Sc}}$, using Bio-Rad's TeSeE ELISA rapid test kit. No sample was positive for scrapie. Fluorescent antibody test for rabies and H\&E staining on samples were carried out for differential diagnosis. These showed no pathological lesions indicative for neurological disease. While our findings do not exclude the presence of scrapie in Jos, we demonstrate that targeted sampling of small ruminants for neuroinfectious disease is feasible in developing countries, pointing to the possibility of implementing such a monitoring scheme in Nigeria to prevent economic losses in small ruminant livestock as scrapie caveats from endemic countries have shown.

\section{Introduction}

Scrapie is a naturally occurring progressive, fatal, infectious, and neurodegenerative disease of sheep and goats that has been recognized for over 250 years. The occurrence of scrapie preceded the recognition of other prion diseases of mammals and so can be referred to as the archetype of prion disorders [1]. It is caused by an infectious misfolded protein, termed prion. Like other TSEs, this misfolding is thought to be caused by conformational change of the normal host prion protein $\left(\mathrm{PrP}^{\mathrm{C}}\right)$ mediated by the presence of the abnormal, misfolded prion protein $\left(\mathrm{PrP}^{\mathrm{Sc}}\right)$. Host $\mathrm{PrP}$ variations are known to play a role in the susceptibility of goats and sheep to scrapie [2-4]. It has been proposed as the root from which bovine spongiform encephalopathy (BSE) emanated [5]. Scrapie is now considered a worldwide disease of small ruminants and currently represents a significant part of the detected transmissible spongiform encephalopathies (TSEs).

The estimated population of sheep and goats in Nigeria is 27 million, and 40.8 million respectively [6]. Sheep are mainly kept for subsistence and religious inclinations while certain goat species are kept for food and high quality leather production [7]. Livestock diseases pose a challenge to this growing economic sector. Scrapie has not been identified so far in Nigeria and research on scrapie and indeed other prion diseases in Nigeria is virgin. However, anecdotal reports of clinical suspicious scrapie and other neurologic trans-boundary diseases abound. Our study aims to establish a basis for an active surveillance programme that primarily addresses the question on the presence of scrapie in Nigeria. This is intended to give an overview of what a wider survey may reveal.

\section{Area Descriptions, Material Studied, Methods, and Techniques}

This study was carried out in Jos, Nigeria, which is a hub of livestock traversing the middle belt and northern parts of the country. We initially planned to sample cases of fallen stock and scrapie suspects from the field where unconfirmed 
TABLE 1: Results of clinical examination and laboratory testing of emergency/casualty slaughter sheep for scrapiefrom Jos, Nigeria.

\begin{tabular}{|c|c|c|c|c|c|c|c|c|c|}
\hline & \multicolumn{8}{|c|}{ Number of sheep per breed and sex } & \multirow{3}{*}{ Tota } \\
\hline & \multicolumn{2}{|c|}{ Yankasa } & \multicolumn{2}{|c|}{ Uda } & \multicolumn{2}{|c|}{ West African dwarf } & \multicolumn{2}{|c|}{ Balami } & \\
\hline & Male & Female & Male & Female & Male & Female & Male & Female & \\
\hline Clinical examination & 250 & 20 & 117 & 13 & 59 & 19 & 17 & 15 & 510 \\
\hline \multicolumn{10}{|l|}{ Changes in mental status, behaviour and activity } \\
\hline Self-Isolation & & & & & & & & & 0 \\
\hline Disobeys herdsman's calls & & & & & & & & & 0 \\
\hline Flighty on approach & & & & & & & & & 0 \\
\hline Hyperalert & 5 & & & & & & & & 5 \\
\hline Increased response to external stimuli & & & & & & & & & 0 \\
\hline Somnolent/dull/vacant gaze/lowered head & 50 & 15 & 11 & 8 & 45 & 11 & 8 & 10 & 150 \\
\hline Often lies down & 16 & 3 & 10 & 2 & 7 & 18 & 11 & 2 & 69 \\
\hline Reduced social interaction & 50 & 15 & 11 & 8 & 45 & 11 & 8 & 10 & 150 \\
\hline Frequent teeth grinding & & & & & & & & & 0 \\
\hline Loss of appetite & 33 & 17 & 11 & 6 & 40 & 11 & 5 & 7 & 130 \\
\hline \multicolumn{10}{|l|}{ Pruritic activities } \\
\hline Increased grooming behaviour & & & & & & & & & 0 \\
\hline Rubs on objects frequently & & & & & & & & & 0 \\
\hline Licking/smacking of mouth & & & & & & & & & 0 \\
\hline Nibble reflex response & & & & & & & & & 0 \\
\hline Rapid tongue extrusion & & & & & & & & & 0 \\
\hline \multicolumn{10}{|l|}{ Poor response to scratch test } \\
\hline Body discolouration & & & & & & & & & 0 \\
\hline \multicolumn{10}{|l|}{ Postural changes } \\
\hline Lowered hind with flexed limbs & & & & & & & & & 0 \\
\hline \multicolumn{10}{|l|}{ Gait changes } \\
\hline Ataxia/discordant movement & & & & & & & & & 0 \\
\hline \multicolumn{10}{|l|}{ Involuntary movements } \\
\hline Head tremors & & & & & & & & & 0 \\
\hline Seizures & & & & & & & & & nil \\
\hline \multicolumn{10}{|l|}{ Weight loss } \\
\hline Weight loss & 57 & 18 & 51 & 12 & 45 & 10 & 15 & 13 & 221 \\
\hline Clinically suspicious & & & & & & & & & 0 \\
\hline
\end{tabular}

reports of scrapie filter in once in a while. This proved difficult due to the uncooperative attitude of livestock owners/herds men and poor records on their animals. We noted that most diseased animals that would match the clinical picture of scrapie ended up in the abattoir for the food chain. Hence, we targeted samples of emergency or casualty slaughtered sheep and goats. We examined sheep and goats brought in for emergency or casualty slaughter at the abattoir for clinical signs of scrapie [8] using the guidelines in the OIE Terrestrial Animal Health Code 2009 [1]. For this study, we defined emergency/casualty slaughter animals as sheep or goats that are brought in for slaughter due to unidentified disease condition or debilitating condition that the owner or veterinarian could not identify or treat successfully. All animals brought in as routine slaughter are not inclusive.

Sampling was from January to August 2011. 510 sheep and 618 goats aged from 12 months to 5 years were clinically examined. Physical appearance and dentition were used to assess the animals age. The clinical examination included the assessment of changes in mental status, behavior and activity, pruritic activities, postural changes, gait changes, involuntary movements, weight loss, and loss of condition. Our criteria for selecting clinical scrapie suspect animals primarily focused on animals that showed pruritis, increased levels of excitement on sudden agitation coupled with ataxia or discordant movement, and signs in other categories we could examine for (see Tables 1 and 2; criteria used for case-definition are indicated by italic figures).

We collected caudal brain stems from clinical suspect animals through the foramen magnum postmortem, using a special harvesting spoon. From each sample, a $1 \mathrm{~g}$ portion and two $2 \mathrm{~g}$ portions of the obex region were cut out. The first $2 \mathrm{~g}$ portion was fixed in a sample bottle in 10 times its volume of formalin. Parts of the fixed portions were used for histological processing and hematoxylin and eosin (H\&E) staining while the remaining part was left for confirmation of rapid test positive samples via immunohistochemistry (IHC) [1]. The second portion was packed in a transport container and stored frozen $-20^{\circ} \mathrm{C}$, likewise the remaining portion of the brain stem. The $2 \mathrm{~g}$ frozen portions were shipped to the NeuroCentre, University of Berne, Switzerland, for scrapie testing. The $1 \mathrm{~g}$ portions were used for rabies diagnosis via indirect fluorescent antibody testing (DFA) technique. 
TABLE 2: Results of clinical examination and laboratory testing of emergency/casualty slaughter goat for scrapie, rabies, and H\&E from Jos, Nigeria.

\begin{tabular}{|c|c|c|c|c|c|c|c|c|c|}
\hline & \multicolumn{8}{|c|}{ Number of goats per breed and sex } & \multirow{3}{*}{ Total } \\
\hline & \multicolumn{2}{|c|}{ Kano brown } & \multicolumn{2}{|c|}{ Sokoto red } & \multicolumn{2}{|c|}{ West African dwarf } & \multicolumn{2}{|c|}{ Sahel/Desert } & \\
\hline & Male & Female & Male & Female & Male & Female & Male & Female & \\
\hline Clinical examination & 200 & 94 & 182 & 5 & 77 & 16 & 20 & 14 & 608 \\
\hline \multicolumn{10}{|l|}{ Changes in behaviour and activity } \\
\hline Self-Isolation & 33 & 12 & 7 & & & 3 & & & 55 \\
\hline Disobeys herdsman's calls & & & & & & & & & 0 \\
\hline Flighty on approach & 37 & 15 & 12 & 5 & 17 & 6 & 3 & 5 & 100 \\
\hline Hyperalert & 10 & 6 & 5 & 4 & 4 & 3 & & & 32 \\
\hline Increased response to external stimuli & 10 & 6 & 5 & 4 & 4 & 3 & & & 32 \\
\hline Somnolent/dull/vacant gaze/lowered head & & & & & & & & & 0 \\
\hline Often lies down & 3 & 1 & & 2 & & 1 & & & 7 \\
\hline Reduced social interaction & 3 & 1 & & 2 & & 2 & & & 7 \\
\hline Frequent teeth grinding & & & & & & & & & 0 \\
\hline Loss of appetite & 45 & 13 & 27 & 5 & 21 & 8 & 5 & 3 & 127 \\
\hline \multicolumn{10}{|l|}{ Pruritic activities } \\
\hline Increased grooming behaviour & 11 & 6 & 5 & 3 & & 5 & & & 29 \\
\hline Rubs on objects frequently & 8 & 21 & 7 & 1 & 5 & 7 & 1 & 1 & 51 \\
\hline Licking/smacking of mouth & 14 & 13 & 5 & & & 2 & & & 34 \\
\hline Nibble reflex response & & & & & & & & & 0 \\
\hline Rapid tongue extrusion & 14 & 13 & 5 & & & 2 & & & 34 \\
\hline Poor response to scratch test & & & & & & & & & 0 \\
\hline Body discolouration & & & & & & & & & 0 \\
\hline \multicolumn{10}{|l|}{ Postural changes } \\
\hline Lowered hind with flexed limbs & 10 & & 6 & 2 & 10 & 2 & 2 & 2 & 35 \\
\hline \multicolumn{10}{|l|}{ Gait changes } \\
\hline Ataxia/discordant movement & 8 & & 7 & 2 & 9 & 2 & 2 & 1 & 31 \\
\hline \multicolumn{10}{|l|}{ Involuntary movements } \\
\hline Head tremors & 7 & & 5 & & & 1 & 1 & 1 & 15 \\
\hline Seizures & & & & & & & & & \\
\hline \multicolumn{10}{|l|}{ Weight loss } \\
\hline Weight loss & 53 & 47 & 71 & 5 & 23 & 15 & 11 & 7 & 238 \\
\hline Clinically suspicious & 8 & & 7 & 2 & 9 & 2 & 2 & 1 & 31 \\
\hline TeSeE ELISA test positive & & & & & & & & & 0 \\
\hline DFA test positive & & & & & & & & & 0 \\
\hline H\&E test result & & & & & & & & & 0 \\
\hline
\end{tabular}

Italic figures denote parameters considered for selecting suspects.

Testing for scrapie was done using the TeSeE ELISA purification kit (Bio-Rad, ref.: 355 1165) and detection kit (Bio-Rad, ref.: 355 1166). This kit employs an immunoenzymatic technique (sandwich format) using two monoclonal antibodies for the detection of $\mathrm{PrP}^{\mathrm{Sc}}$. While the type of these monoclonal antibodies is subject to industrial secret, its capacity to detect classical scrapie in a range of sheep with different PrP genotypes has been shown [9]. However, similar data for goats are still missing. This test was carried out according to the manufacturers guide after sample preparation/clarification and proteinase $\mathrm{K}$ digestion. Any positive sample would have been confirmed by IHC $\operatorname{PrP}^{\mathrm{Sc}}$ staining method using monoclonal antibody F99 (VMRD, epitope at the prion protein C-terminal: QYQRES) on tissue sections prepared from formalin fixed tissues [1]. Scrapie positive and negative controls were set up alongside kit controls during testing. We did not determine the PrP genotype of the clinical suspicious goat samples collected before screening for scrapie because of limitations in funding.

\section{Results and Discussion}

By clinical examination, we did not identify any scrapie suspect sheep (Table 1) but identified in total 31 (5.10\%) of 608 goats as scrapie suspicious (Table 2). The absence of clinically suspicious sheep may be attributed to the religious importance attached to sheep by the predominant tribe (Fulani) that breed sheep as compared to goats in the study area. Apparently healthy sheep with mild/visible debilitation that could be overlooked were brought in for emergency slaughter unlike goats that were basically sold and slaughtered for food and leather. Hence on examination and in the absence of pruritis and gait problems we classed such sheep as clinically unsuspicious though they were examined for some other 
signs (Table 1). We note that nonoptimal or ideal ante mortem livestock handling practices by owners/butchers prior to slaughter may have elicited increased agitation. These could have led to poor clinical examination of some sheep and goats which may have given rise to appearances of seemingly clinical suspects.

Clinical examination revealed 31 goats with neurological signs indicative for scrapie. However, this suspect could not be confirmed by laboratory testing. This underlines the limitations associated with clinical examination of livestock for prion disease [10], as clinical signs in scrapie are not very specific.

The PrP genotypes of local breeds of goats and sheep in Nigeria are yet to be determined. As the nature and proportion of PrP genotypes in the sheep and goat population in Nigeria remain unknown, it is possible that previously unnoticed PrP genotypes are present that interfere with the performance of diagnostic tests applied but this remains unknown. Additional studies are required to fill this gap of knowledge. For differential diagnosis, it may be worthwhile to mention that we did not specifically examine nor sample target animals for other diseases like listeriosis, tetanus, brain worm (Cerebrospinal Nematodiasis), caprine arthritis, and encephalities [11] that may have given differential diagnosis viz a vis neurological diseases of small ruminants. We only carried out rabies diagnosis and H\&E staining because these tests can be done using the same samples collected for scrapie testing. None of the samples tested positive for rabies, ruling out rabies as an underlying neurological disease. The H\&E pictures did not reveal any TSE pathological conditions which appear as characteristic vacuolation affecting the neuropil with an anatomical and bilateral symmetrical distribution [12]. This means that the observed seeming neurological conditions that we aligned with discordant movement or other abnormal gait conditions may be results of other, not neurological disease. Pruritis may have been results of early infection with dermatophilic diseases that were not visible and had not degenerated to loss or discolouration of fur. Considering that we used a targeted sampling method which excluded other surveillance streams, a small sample size and area of study in relation to the sheep and goat population of the country, our findings should not be seen as the absence of scrapie in the study area or extrapolated as an extended picture of the countries scrapie status. We have only shown that sheep and goat that we considered clinical suspect cases alongside anecdotal reports were not positive for scrapie using TeSeE ELISA by Bio-Rad testing and the criteria we used for clinical suspicion may have been misinterpreted or not properly applied.

Difficulties and challenges that may be faced when establishing an active surveillance in Nigeria are some we came across in this study: the nomadic nature of livestock farming, poor enlightenment, poor or inexistent records of livestock by owners, no compensatory benefit systems in place for livestock owners and inadequate veterinary services. These affect the quality of demographic data obtained and ease of diagnosis. Clinical suspect animals are not being officially reported for testing, hence an alternative means is to actively search for animals with neurological signs as we did until the challenges confronting the proper setup of a surveillance system for
TSE are addressed; enlightenment programs for farmers and animal handlers, personnel training on prion diseases and proper laboratory diagnostic setups in place by government agencies concerned.

This study lays the groundwork for an efficient and costeffective surveillance of scrapie and other neuroinfectious diseases of livestock in resource-limited countries, such as $\mathrm{Ni}$ geria. This we have demonstrated by systematic ante mortem inspection of emergency and casualty slaughter animals that would have identified neurologically diseased small ruminant livestock in a relatively high proportion. We advocate the use of our findings as a platform for the setup of an active surveillance for scrapie and other animal TSEs in Nigeria so as to determine the scrapie status for economic reasons.

\section{Conflict of Interests}

The authors declare that there is no conflict of interests in this research. The authors do not have any form of financial relation with the commercial identities mentioned in this paper.

\section{Acknowledgment}

The authors thank the Management of National Veterinary Research Institute Nigeria for permitting and supporting this study. They also thank the management and staff of NeuroCentre, National and OIE Reference Laboratories for BSE and Scrapie, Division of Experimental Clinical Research, Vetsuisse Faculty, University of Berne, Berne, Switzerland, for personnel training and laboratory analysis of samples. The NeuroCentre is partially funded by the Swiss Federal Veterinary Office.

\section{References}

[1] "NB: version adopted by World Assembly of Delegates of the OIE in May, 2009," in OIE Terrestrial Manual. Manual For Diagnostic Tests and Vaccines For Terrestrial Animals 2009, OIE Chapter 2.7.13. Scrapie, pp. 1-10, Blackwell Publishing, Ames, Iowa, USA, 2010.

[2] P. B. G. M. Belt, I. H. Muileman, B. E. C. Schreuder, J. Bos-de Ruijter, A. L. J. Gielkens, and M. A. Smits, "Identification of five allelic variants of the sheep $\operatorname{PrP}$ gene and their association with natural scrapie," Journal of General Virology, vol. 76, no. 3, pp. 509-517, 1995.

[3] N. Hunter, "Natural scrapie in a closed flock of Cheviot sheep occurs only in specific PrP genotypes," Archives of Virology, vol. 141 , no. 5, pp. 809-824, 1996.

[4] M. Baylis, F. Houston, W. Goldmann, N. Hunter, and A. R. McLean, "The signature of scrapie: differences in the PrP genotype profile of scrapie-affected and scrapie-free UK sheep flocks," Proceedings of the Royal Society B, vol. 267, no. 1457, pp. 2029-2035, 2000.

[5] J. W. Wilesmith, J. B. Ryan, and M. J. Atkinson, "Bovine spongiform encephalopathy: epidemiological studies on the origin," Veterinary Record, vol. 128, no. 9, pp. 199-203, 1991.

[6] L. H. Lombin, "Report on the internet," 2007, http://www. africanagricultureblog.com/2007/12/nigeria-has-16-millioncattle.html 2007. 
[7] R. Blench, "Traditional livestock breeds: geographical distribution and dynamics in relation to the ecology of West Africa," Working paper 122 presented to the Nigerian National Livestock Resource Survey (NNLRS) with Resource Inventory and Management (RIM), October 1999.

[8] C. Fast and M. H. Groschup, "Classical and atypical scrapie in sheep and goat," in Prions and Diseases Vol. 2, Animals, Humans and the Environment, W. Q, Zou, and P. Gambetti, Eds., Springer, New York, NY, USA, 2013.

[9] European Food Safety Authority, "Evaluation of rapid post mortem TSE tests intended for small ruminants," EFSA Journal, vol. 31, pp. 1-17, 2005.

[10] T. Konold, S. K. Sivam, J. Ryan, S. Gubbins, R. Laven, and M. J. H. Howe, "Analysis of clinical signs associated with bovine spongiform encephalopathy in casualty slaughter cattle," Veterinary Journal, vol. 171, no. 3, pp. 438-444, 2006.

[11] E. Maurer, C. Botteron, F. Eherensperger et al., "Swiss scrapie surveillance. I. Clinical aspects of neurological diseases in sheep and goats," Schweizer Archiv für Tierheilkunde, vol. 147, no. 10, pp. 425-433, 2005.

[12] TSE EU Community Reference Laboratory (TSE EU CRF), "Neuropathology: confirmatory diagnosis of transmissible spongiform encephalopathies (TSE) in cattle and sheep," TC: Diagnosis1.doc/MMS3, 2004. 

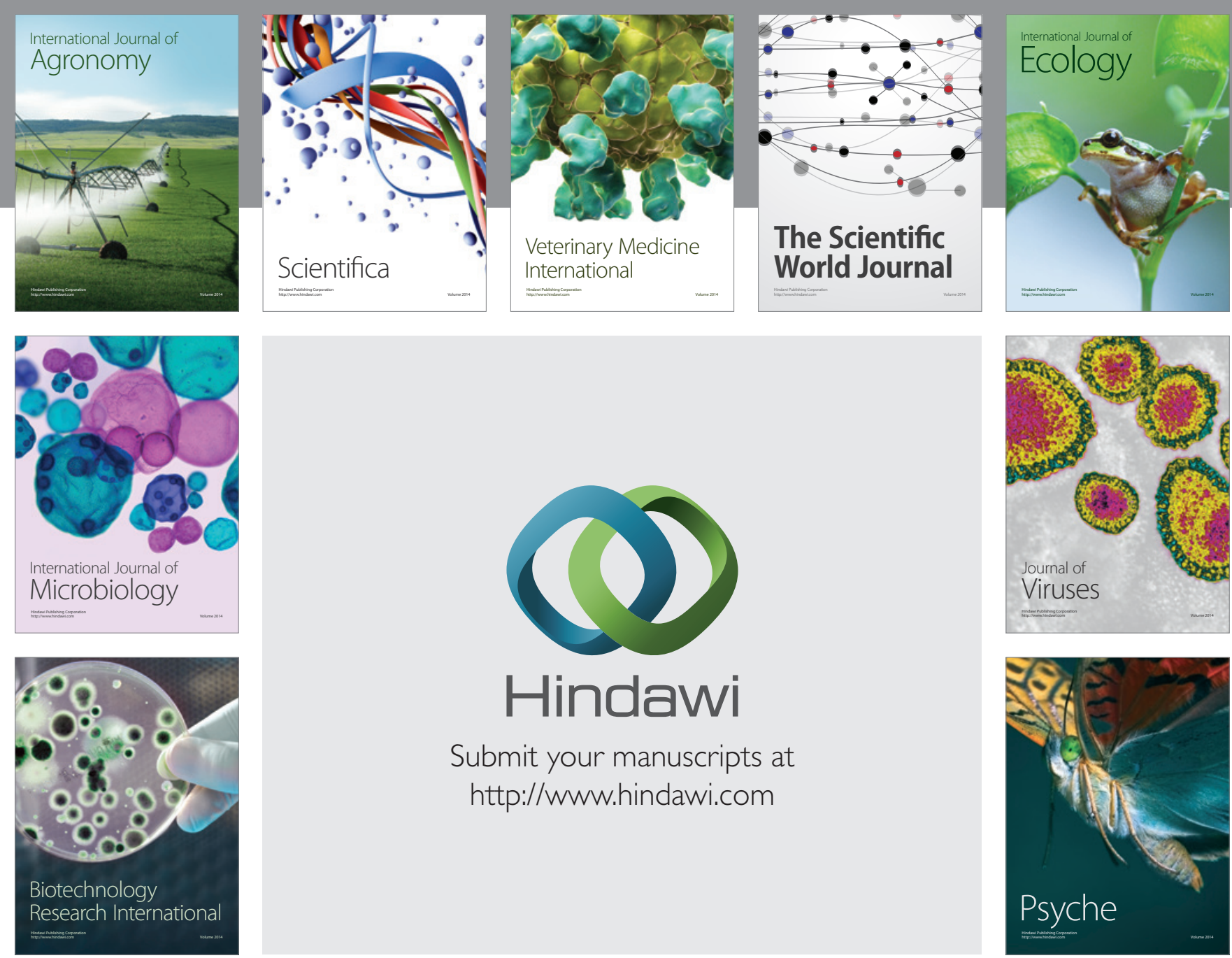

Submit your manuscripts at http://www.hindawi.com
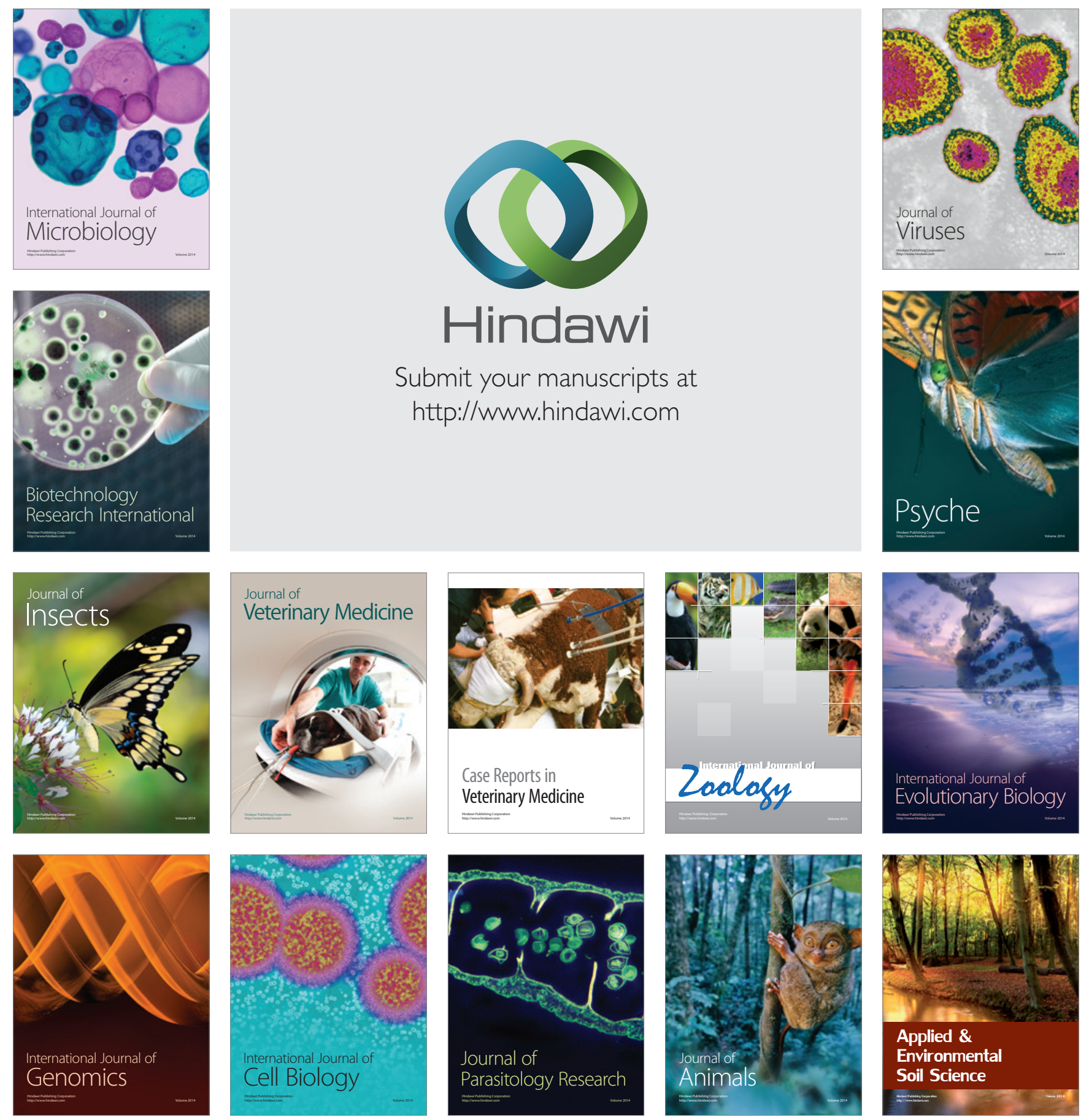